\title{
The Growth Mechanism of Lithium Dendrites and its Coupling to Mechanical Stress
}

\author{
Julian Becherer, Dominik Kramer*, and Reiner Mönig \\ Institute for Applied Materials, Karlsruhe Institute of Technology, \\ Hermann-von-Helmholtz-Platz 1, 76344 Eggenstein-Leopoldshafen, Germany \\ * Corresponding author: dominik.kramer@kit.edu
}

\begin{abstract}
Operando high-resolution light microscopy with extended depth of field is used to observe large regions of an electrode during electrodeposition of lithium. The analysis of the morphology of the evolving deposit reveals that besides electrochemistry, mechanics and crystalline defects play a major role in the growth mechanism. Based on the findings, a growth mechanism is proposed that involves the diffusion of lithium atoms from the lithium surface into grain boundaries and the insertion into crystalline defects in the metal. Crystalline defects are a result of plastic deformation and hence mechanical stimulation augments the insertion of lithium.
\end{abstract}

\section{Introduction}

Lithium metal is the ideal anode material to improve the energy density of batteries compared to the state-of-the-art lithium-ion batteries. However, the commercialization of lithium metal cells is impeded by safety and reliability threats associated with the growth of so-called dendrites during the electrodeposition of lithium, i.e. during the charging of such cells. Dendrites are relevant not only for lithium metal batteries, but also for today's lithium-ion batteries where they will grow at fast charging rates, especially at low temperature. The mechanisms of the deposition and dissolution of lithium are still under debate. Several recent reviews point out the importance of new operando characterization techniques for a better understanding of these mechanisms. ${ }^{[1-5]}$ Nishikawa et al. ${ }^{[6,7]}$ found a linear relationship between the length of the deposits and the square root of time in their study of the growth rate of electrodeposited lithium. They attributed this behavior to the ionic mass transfer within the electrolyte. They only observed lithium bushes, i.e. agglomerates of lithium dendrites and mentioned that it would be beneficial to be able to observe individual dendrites. Several experimental studies, often at higher resolution, observed a growth outside of the tip region of deposits, ${ }^{[8-15]}$ which is in contradiction with growth models based on ionic mass transfer limitations in the electrolyte. In previous operando investigations of our group, the lithium insertion into the crystal lattice at defects such as kinks was identified as dominating growth mechanism. ${ }^{[12-15]}$ Our most recent 
study showed that this mechanism is important at all rates from $-0.05 \mathrm{~mA} \mathrm{~cm}^{-2}$ to $-100 \mathrm{~mA} \mathrm{~cm}^{-2}$, and it even occurs during ionic depletion of the electrolyte. ${ }^{[15]}$ For a cell with an area specific capacity of $3 \mathrm{mAh} \mathrm{cm}^{-2}$, these rates would correspond to C-rates from $\mathrm{C} / 60$ to $33 \mathrm{C}$ and hence all rates that are relevant for practical battery applications. Similar to our observations, Rulev et al. ${ }^{[16]}$ observed the insertion at grain boundaries (GBs) and a growth from the "root" of large lithium grains when lithium was electrodeposited onto a lithium foil.

On several scales, mechanical effects play an important role in the formation of lithium structures. On the cell level, stack pressure has been reported to be a decisive parameter for the suppression of dendrites. ${ }^{[17-20]}$ The displacement of an individual component (e.g. separator) within a cell can damage the solid-electrolyte interphase (SEI) and trigger the growth of fastgrowing bushes. ${ }^{[15]}$ The mechanical properties of the SEI have been often associated with the onset of dendritic growth. ${ }^{[21,22]}$ Even at the lowest scales, growth models exist where the formation of dendrites is caused by mechanical stresses. ${ }^{[8,11]}$

Metal deposition results in adatoms on the surface that diffuse, form dimers, and evolve into islands and mounds. ${ }^{[23]}$ These islands can coalesce and form polycrystalline films or can transform into protrusions. This might result in needles. ${ }^{[14,24]}$ Growth very much depends on atomistic diffusion barriers, including the step-edge (Ehrlich-Schwoebel) barrier. ${ }^{[25]}$ Moreover, crystalline defects play a major role: Compared to an adatom on the surface, which has only a few nearest neighboring metal atoms, an atom in a GB has more bonds to other atoms, which is energetically favorable. From the theory of thin film growth it is known that due to a difference in the chemical potentials, there is a thermodynamic driving force for adatoms to diffuse from surfaces into GBs. ${ }^{[26,27]}$ In addition to the energetics that drives the transport of adatoms into GBs and from there into crystal lattice sites, GBs are also kinetically favored compared to the bulk because surface and GB diffusion are significantly faster than diffusion through the bulk. So far, the growth of lithium needles and kinked structures, which are the building blocks of many lithium deposits, is not fully understood. In this work, we focus on the growth of these kinked needles using optimized operando light microscopy. This involves fast focus stacking and the observation of large electrode areas $\left(\sim 0.5 \mathrm{~mm}^{2}\right)$ close to the physical resolution limit of light. In these large representative electrode areas, the influence of different shapes on the growth velocity of individual structures is analyzed. The results indicate that geometrical constraints and resulting stresses have a strong influence on the growth velocity.

\section{Results}

Before each experiment, a dense film of lithium was electrodeposited onto a copper substrate (for details see experimental section and our previous publication ${ }^{[15]}$ ). In this work, we study 
the electrodeposition of lithium at $-0.05 \mathrm{~mA} \mathrm{~cm}^{-2}$ onto this lithium film. Additional data for $-0.5 \mathrm{~mA} \mathrm{~cm}^{-2}$ can be found in the supporting information. During the experiments, many needles grow on the electrode. They evolve in length and some form kinks and generally grow as described before. ${ }^{[15]}$ At later stages of needle growth, it becomes evident that certain structures grow particularly fast. These structures with multiple kinks are attached to electrode at both ends and from now on will be referred to as loops.
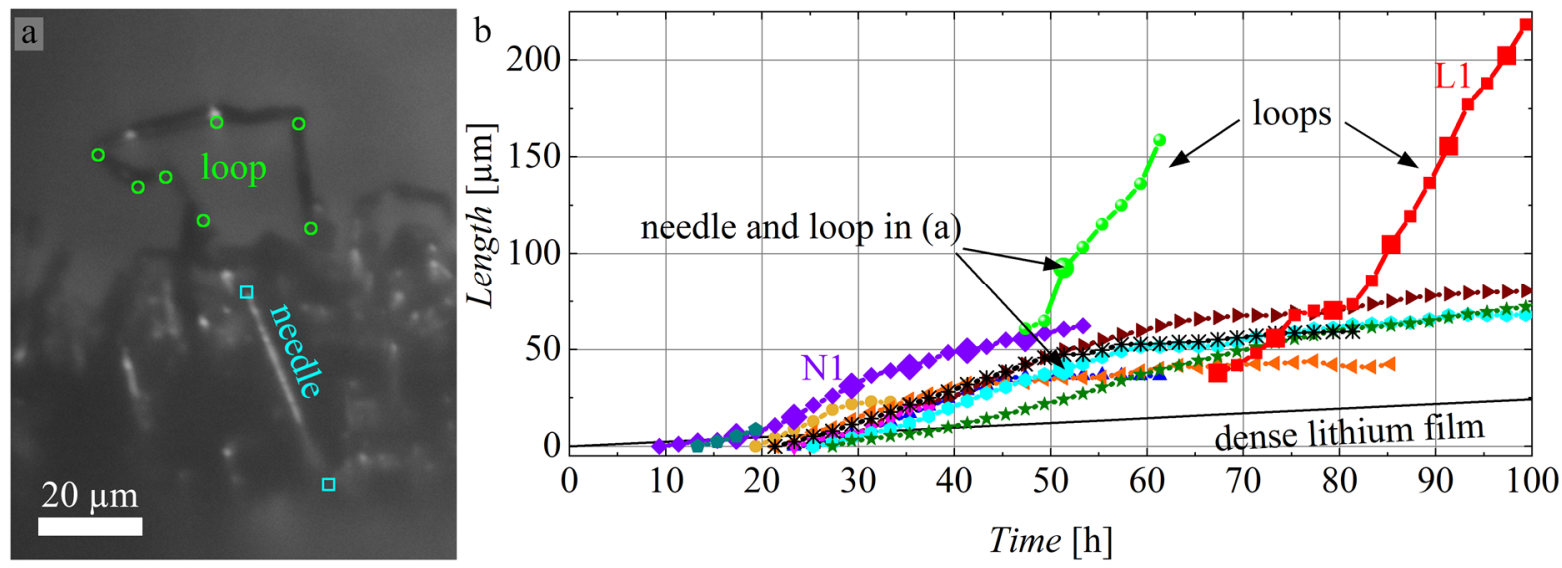

Figure 1. (a) A needle and a loop after approximately $51 \mathrm{~h}$ deposition at $-0.05 \mathrm{~mA} \mathrm{~cm}^{-2}$. (b) The lengths of ten needles and two loops (represented by different colors) during the lithium electrodeposition at $-0.05 \mathrm{~mA} \mathrm{~cm}^{-2}$. The black line is the calculated thickness of a dense lithium film. Large symbols for the needle N1 and the loop L1 indicate when the images in Figure 2 and 3 were recorded.

Figure 1a shows an exemplary needle and a loop. The cyan squares indicate the base where the needle nucleated and the tip of the needle (cyan plot in Figure 1b). The green circles highlight all visible kinks of the loop (green plot in Figure 1b). Figure 1b shows the evolution of the lengths of ten arbitrarily chosen needles and two loops. The total length of each loop is the sum of the lengths of all visible segments between neighboring kinks calculated from 3D data based on the image stacks. The segments at the base of loops are typically covered by other lithium deposits and hence are not visible. Therefore, the length of the loops shown in Figure $1 \mathrm{~b}$ is an underestimation of the actual length. The black line in Figure $1 \mathrm{~b}$ is the calculated thickness of a dense lithium film deposited at the same current density. The growth of both, needles and loops, is considerably faster than the growth of a dense film.
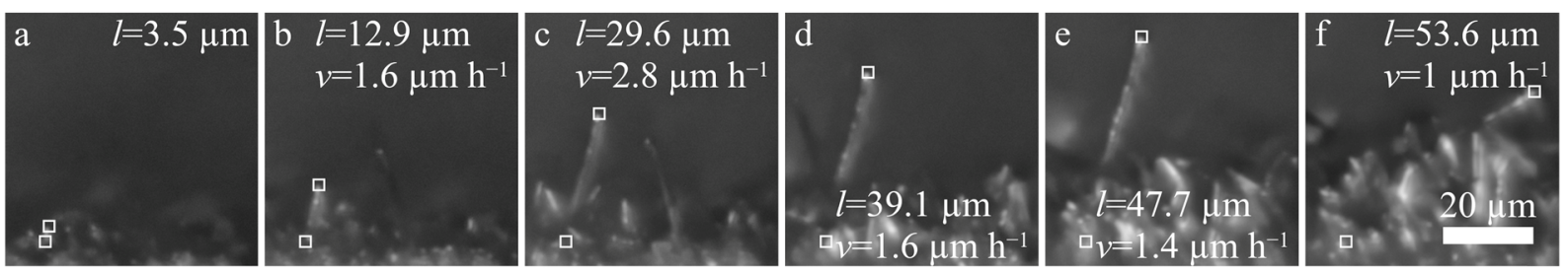

Figure 2. (a) Needle N1 after nucleation. (b-f) 6 hours of deposition at $-0.05 \mathrm{~mA} \mathrm{~cm} \mathrm{~cm}^{-2}$ between the images. White squares in the lower left of each image marks the position of nucleation, while the second square marks the tip of the needle. The larger symbols in the N1-plot of Figure $1 \mathrm{~b}$ indicate when the images (a-f) were recorded. 
The observed needles nucleated between 9 and 27 hours after the start of the experiment. After nucleation, they typically exhibit accelerating growth that later diminishes and eventually the growth stops (e.g. the orange triangles in Figure 1b). The total length of needles that stopped varies between $\sim 20 \mu \mathrm{m}$ and $\sim 80 \mu \mathrm{m}$. The growth of the needle N1 is shown in Figure 2 and the supporting video SV1, where a growth from the base of the needle is clearly visible. This base growth was typically observed for needles without kinks. The velocities given in Figure $2 \mathrm{~b}-\mathrm{f}$ increase initially and decrease later. Between Figure 2e and f, a kink appeared and the distance between base and tip does not represent the length of the needle anymore. Kinking of some needles and the blocking of the view onto the tip caused by other deposits complicated the length measurements at later times. In spite of these experimental difficulties, the observed deceleration of the growth as seen in Figure 1b, is a real and general trend.
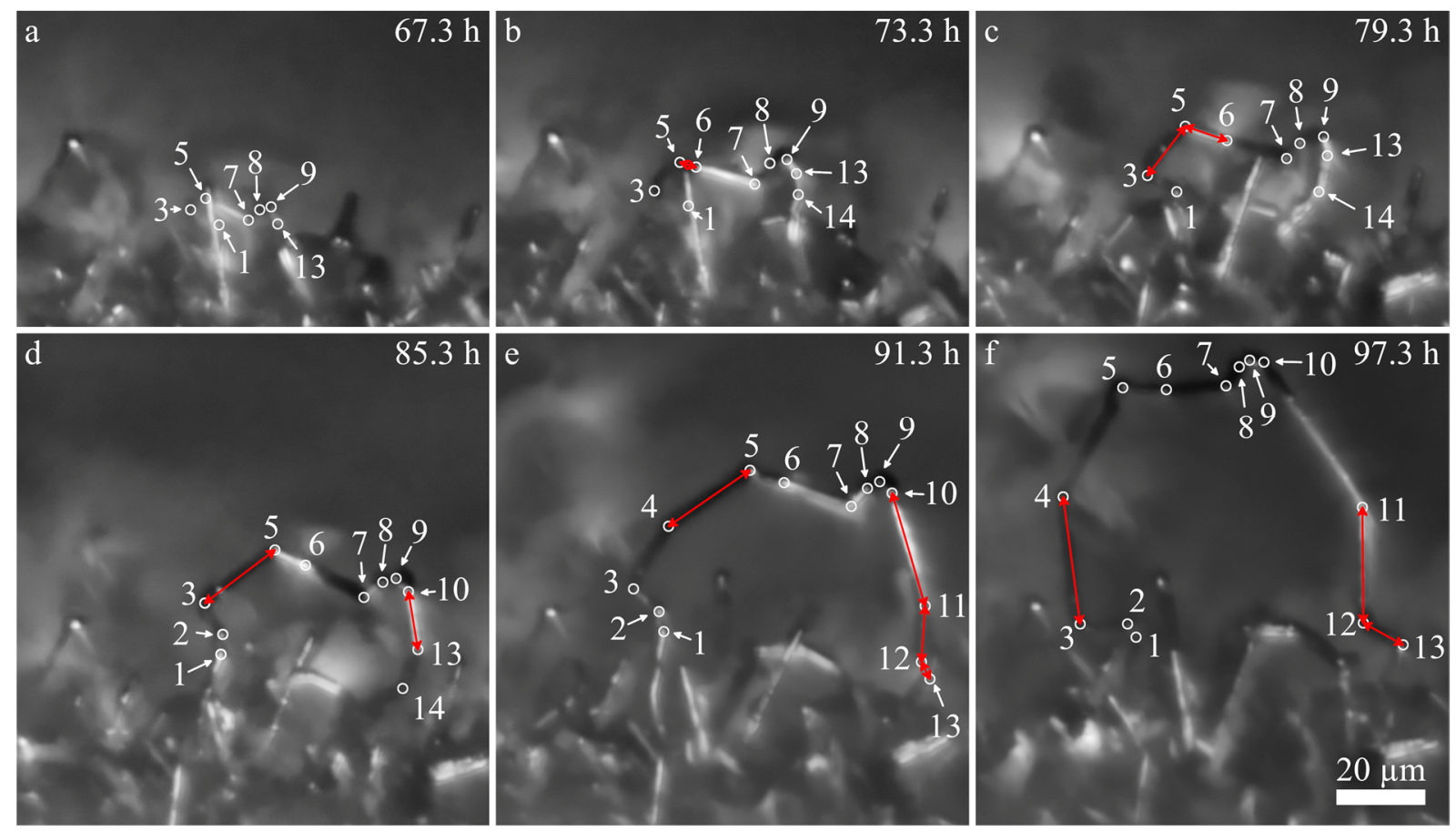

Figure 3. (a-f) Growth of the lithium loop $\mathrm{L} 1$ at $-0.05 \mathrm{~mA} \mathrm{~cm}^{-2}$ with 6 hours of deposition between the images. White circles indicate kinks of the loop. The number of kinks increases over time. Kink 14 is not visible in all of the images as other needles block the view. The larger symbols in the L1-plot of Figure $1 \mathrm{~b}$ indicate when the images (a-f) were taken.

Compared to needles, loops appear later in the experiments. In Figure 1b, it becomes apparent that loops can grow significantly faster than the needles. The growth of the loop L1 (red plot) is shown in detail in Figure 3 and the supporting video SV2. In Figure 3, all visible kinks are marked by white circles and numbered. From the images, it becomes clear that the segments grow at different speeds and individual segments dominate the growth. The segments that dominate (red arrows in Figure 3) can change over time. The fastest growing segments are either next to newly formed kinks (kink 6 in b, kink 10 in d, and kink 4, 11, and 12 in e) or next to segments that grew fast beforehand (segment 3-5 in c and segment 3-4 in f). The segments 
between kink 7 and 9 hardly grow during the $30 \mathrm{~h}$ of deposition shown in Figure 3, while some segments reached growth velocities beyond $4 \mu \mathrm{m} \mathrm{h}^{-1}$ (measured for $2 \mathrm{~h}$ intervals), which is significantly faster than the fastest velocities observed for individual needles (up to $3 \mu \mathrm{m} \mathrm{h}^{-1}$ ). During deposition, the number of kinks increases. Freshly formed kinks appear either next to another kink (kink 2, 6, and 10) or between two kinks (4, 11, and 12).

The crystal orientations of the segments of a loop that grew during the deposition shown in Figure 1, 2, and 3 were analyzed using electron backscatter diffraction (EBSD) in the scanning electron microscope (SEM). Although the EBSD patterns are weak, as it is expected for lithium, they clearly show that the crystal orientation of each segment is constant but changes at kinks, i.e. GBs are present at the kinks. The supporting video SV3 (see also the explanatory note in the supporting information) shows the EBSD patterns for various measurement points. As previously reported $^{[12,15]}$, the lithium insertion occurs at the kinks and hence at GBs.

Figure 4 shows SEM images of kinked lithium structures after $100 \mathrm{~h}$ deposition at $-0.05 \mathrm{~mA} \mathrm{~cm}^{-2}$. Figure $4 \mathrm{a}$ contains a small loop where both ends are connected to the surface. It shows an abrupt change in diameter on its right limb. The end of the loop at the bottom of the image appears to be almost parallel to the substrate. Figure $4 \mathrm{~b}$ shows a strongly kinked needle containing segments with different contrast and different diameter. Their surface structure and faceting seems to vary. A striking feature are the periodic fine rings perpendicular to the growth direction. Figure $4 \mathrm{a}$ and $\mathrm{b}$ show dark regions. The image of Figure $4 \mathrm{c}$ was taken at low accelerating voltage (more surface sensitivity) and shows these dark regions more clearly. According to energy dispersive X-ray (EDX) analysis (see supporting Figure S1), these dark regions contain more oxygen and more carbon than the surrounding surfaces.
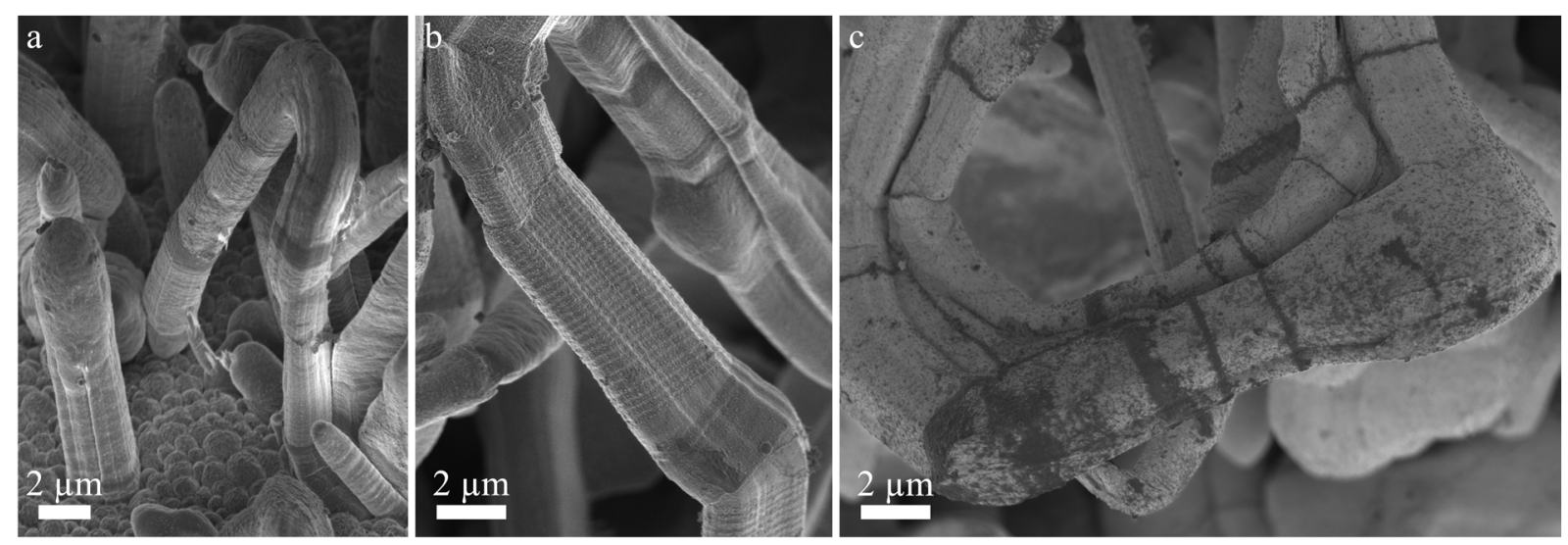

Figure 4. Lithium deposits formed at a current density of $-0.05 \mathrm{~mA} \mathrm{~cm}^{-2}$. (a) A small loop and its connection to the substrate. (b) Needles with multiple kinks and defects at the kinks. The different segments exhibit different surface structures. (c) Image of a loop recorded at low acceleration voltage (high surface sensitivity). The images were taken at acceleration voltages of (a) $1.5 \mathrm{kV}$, (b) $1 \mathrm{kV}$, and (c) $0.3 \mathrm{kV}$. 
Similar observations as descried for the deposition at $-0.05 \mathrm{~mA} \mathrm{~cm}^{-2}$ were made for a ten times

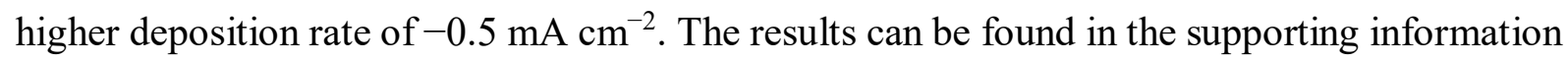
(Figure S2 and S3) The general growth behavior was the same. The following minor differences that were observed: (i) the individual needles grow significantly shorter; (ii) loops tend to kink more frequently, which results mostly in shorter segments (see supporting video SV4 and Figure S3); (iii) spherical bulges were regularly observed at kinks (inset Figure S3d); (iv) an acceleration in the growth rate of the needles was not observed after nucleation; (v) the needles and loops grew significantly faster compared to Figure $1 \mathrm{~b}$, but slower than expected given the ten times higher rate.

\section{Discussion}

The growth of individual structures, needles and loops, during the electrodeposition of lithium was investigated by operando light microscopy. Root growth and growth at kinks were observed as reported before. ${ }^{[8,12,15]}$ Growth by insertion into these locations is not in agreement with models based on the ionic mass transport limitations of the electrolyte that result in growth at the tips. Examples of such models are those valid in diluted solutions at high $\operatorname{rate}^{[28]}$ and in polymers electrolytes ${ }^{[29]}$.

The observed growth kinetics does not correspond to the change in ionic concentration that is expected for a galvanostatic experiment. Based on light microscopy, Nishikawa et al. ${ }^{[6,7]}$ concluded that the ionic transport limits the growth because the length of the deposit scaled with the square-root of time $(\sqrt{t})$ as is characteristic for diffusion. However, other mechanisms like a random-walk movement of a tip of a kinking structure can result in a very similar time dependence. ${ }^{[13]}$ For individual needles, we did not observe such a $\sqrt{t}$ dependence; the length vs. time curve of a needle often has an inflection point (i.e. a maximum growth rate). Given the kinked geometry of needles, loops, and bushes and the location of insertion sites, we also do not see a physical reason to assume that there is a diffusional transport limitation in the solution that causes a simple $\sqrt{t}$ law (for a more detailed discussion, see supplement section A3). SEI thicknesses are often assumed to grow with $\sqrt{t}$, but at the active insertion points the lithium surface is renewed continuously, so it is not expected that the SEI thickness grows with a $\sqrt{t}$ law. Therefore, the apparent $\sqrt{t}$ dependence might be rather a coincidence than a diffusionrelated effect. For all battery relevant deposition rates, ionic transport limitations in the electrolyte anyway only play a minor role compared to the defect related insertion mechanism. ${ }^{[15]}$ GBs were observed at kinks (supporting video SV3 and explanatory note in the supporting information). They consist of crystalline defects and contain vacancies. High-angle boundaries can be described by amorphous regions and low angle boundaries are often treated as arrays of 
dislocations. For lithium, room temperature is approximately $0.65 \cdot T_{\mathrm{m}}$ (melting temperature in K). Typical deformation mechanism maps of metals ${ }^{[30]}$ show that at such high homologous temperatures, deformation is not solely based on dislocation glide. In addition, three diffusive processes are active already at lower stresses: These are (i) GB diffusion (in bulk materials known as Coble creep) and (ii) dislocation creep (climb). Since the deposits have very small dimensions (Figure 4) and hence exhibit high surface to volume ratios, (iii) surface diffusion may also contribute to the deformation and growth of segments. It may be expected that at room temperature diffusion along the surface is even faster than the already facile GB diffusion. A coupling of the transport paths is very likely. A similar coupling between surface and interface diffusion (interfacial Coble creep) has already been identified for a combination of lithium and a mixed ionic-electronic conductor. ${ }^{[31]}$ From the literature, it is known that most metal atoms can diffuse from the surface into and out of GBs and thereby cause mechanical stresses. ${ }^{[26,32,33]}$ This has been observed on metal thin films on rigid substrates. During physical vapor deposition of metallic thin films, adatoms migrate into the GBs and cause compressive stresses. ${ }^{[26,27,33]}$ For thin films on substrates, compressive stress arises due to the mechanical constraints of the growing film that is rigidly attached to a substrate. This is different in the kinked needles where grains are arranged sequentially and are free to elongate by displacing their neighbors. Our deposits contain GBs between their segments: EBSD shows that segments that are separated by kinks have different crystal orientations (supporting video SV3). In contrast to our EBSD measurements, high-resolution transmission electron microscopy (TEM) ${ }^{[34]}$ and electron diffraction $^{[35]}$ indicate that kinked structures can be single crystalline. Although we have not found neighboring segments with the same crystal orientation, we cannot exclude that also in our samples kinks exist that are not GBs. Based on our EBSD results, the observation of elongating segments (Figure 2 and Figure 3, cf. Steiger et al. ${ }^{[12]}$ ) suggests that growth takes place by lithium insertion into these GBs. During electrodeposition, lithium adatoms are deposited onto the substrate and onto the segments of the needles. On the surface of the segments, there are few defects (e.g. islands with ledges) and the probability for adsorption (i.e. crystal growth) is low. Therefore, adatoms remain mobile and can diffuse towards a GB (Figure 5). Inside the GB, atoms have a higher coordination (number of neighbors) than on the surface and adatom insertion into the GB is expected to be energetically favorable. Since diffusion is facile there as well, the lithium atom can move along the GB. For example, such a coupled diffusion path of surface and GB diffusion has been identified as a stress relaxation mechanism for copper films at high temperature. ${ }^{[32]}$ The GB itself consists of crystalline defects, and additional crystalline defects (plasticity) are introduced into the GBs and the segments by mechanical stresses. Typical defects are dislocations, which are line-like distortions of the crystalline lattice. Along the line of 
a dislocation, diffusion is enhanced (pipe diffusion) compared to the undistorted bulk. Atoms may move away from the GB into dislocations or vacancies (Figure 5) and thereby enter the bulk. Here again, the coordination of the lithium atom is higher and hence the insertion into a defect is energetically favorable. The insertion of atoms into crystalline defects contributes to the healing of the defects and causes a growth of a segment, i.e. for the case of an edge dislocation the insertion introduces climb. During climb, the atom is inserted at the edge of the extra half-plane and the dislocation displaces. With this step, the crystalline lattice as a whole shifts/grows perpendicular to the extra half-plane by a fraction of a Burgers vector (cf. supporting information Figure S4). Repeated atom insertion of this type leads to crystal growth along the Burges vector. For a body-centered cubic (bcc) lattice, the Burgers vector lies along the $\langle 111\rangle$ direction. This growth direction has been observed and reported as predominant one in the literature. ${ }^{[34-36]}$ Maybe instead of the minimization of the surface energy $y^{[34,37]}$, the insertion atoms into defects determines the growth direction. For single-crystalline whiskers of lithium and tin, growth has been described by the climb of prismatic dislocation loops (Bardeen-Herring climb source). ${ }^{[35,38]}$ This mechanism is based on climbing dislocations as well and may also be relevant here. In general, defects act as sinks for lithium atoms and thereby continuously heal and reform during growth. Most likely, this healing during ongoing deposition is different from the well-known and more static healing/annealing of defects while no excess atoms are present.

Bulk lithium
Grain boundary
SEI on bulk lithium
SEI on grain boundary
$\odot \quad$ Li $^{+}$-ion
$\quad \quad$ Li adatom/lattice atom
$\square \quad$ Vacancy
$\mathrm{S} \quad$ Screw dislocation
$\perp \quad$ Edge dislocation

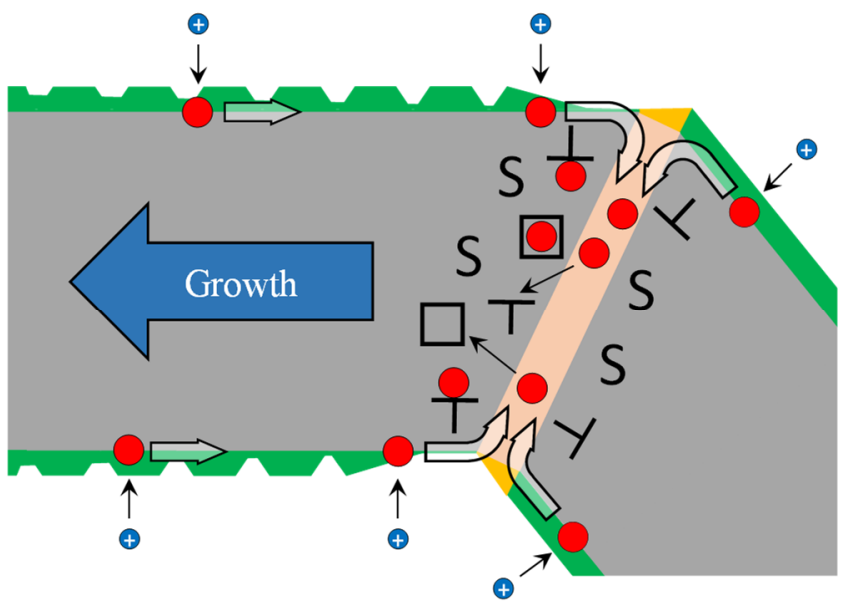

Figure 5. Schematic illustration of lithium insertion into a kinked deposit. Adatoms diffuse along the surface into grain boundaries where they then move into crystalline defects and cause growth of the segment on the left side.

It can be expected that due to geometrical reasons and the resulting stress distribution, the number of defects differs between neighboring segments. Growth may be faster in one of the two segments (e.g. the left segment in Figure 5). Instead of healing, dislocations (defects) can minimize the energy by building regular arrays i.e. low-angle grain boundaries. In polycrystalline 
bulk materials, this is known as subgrain formation and happens during dynamic recrystallization. In loops, similar processes could lead to the formation of additional kinks. Several freshly formed kinks can be found in Figure 3 (e.g. kink 4 formed between Figure 3d and e).

The crystalline defects that act as insertion sites might facilitate unidirectional growth. The observations clearly show that segments elongate, but do not grow in their diameter. The high homologous temperature, which enables the facile diffusion of atoms on the faceted surfaces, results in a facetted shape of the segments that is determined by the minimization of surface energy (Wulff construction ${ }^{[39]}$ ). Steps and irregularities at the surfaces that can be created by dislocation activity (i.e. lateral growth) may decay. Their atoms may diffuse along the surface to the GB where they are reinserted. In addition, the influence of the mechanically stiff SEI might be of importance in the way that it mechanically restricts lateral growth.

Beside the crystalline effects favoring the insertion at the base and at kinks, the SEI will most likely play an important role. Since it is known that even the crystal orientation of lithium influences the SEI, ${ }^{[40]}$ it is very likely that the SEI on a GB differs significantly from the SEI on bulk lithium. The different surfaces we have found at kinks or in their vicinity with surface sensitive SEM images at low acceleration voltages (Figure $4 \mathrm{~b}$ and c) and the differences in the chemical composition (Figure S1) support the concept of an altered SEI at the insertion locations. The higher concentration of carbon and oxygen in Figure S1 may indicate a higher content of crystalline $\mathrm{Li}_{2} \mathrm{CO}_{3}$ grains within the SEI on the grain boundaries. $\mathrm{Li}_{2} \mathrm{CO}_{3}$ was found in the SEI with a similar electrolyte by high-resolution TEM. ${ }^{[34]}$ This most likely affects the transport of lithium-ions through the SEI in these regions. Also important seems the fact that the SEI has to rupture frequently in locations where growth occurs and thus the transport to the insertion sites can occur without SEI or through a thin and defective SEI. For example, the periodic rings perpendicular to the growth direction shown in Figure $4 \mathrm{~b}$ could be caused by repetitive changes (e.g. cracking) of the SEI during growth. Slower growth may not result in such patterns since the SEI has sufficient time to recover. The cracking and healing of the SEI during growth of lithium whiskers was observed by Yang et al. ${ }^{[35]}$ in a TEM study where $\mathrm{Li}_{2} \mathrm{CO}_{3}$ was used as solid electrolyte. They conclude that cracking dominates at high rates, resulting in the axial growth of lithium whiskers, while self-healing of cracks resulted in the growth of spherical particles at lower rates. Our observations show that, for the larger currents, bulges often form at kinks (inset Figure S3d). In this case, the transport inside the GB might not be fast enough to accommodate the flux of adatoms from the surface. Often bulges appear at the end of short segments (Figure S3) in the proximity of other bulges. Therefore, either a short segment is a consequence of the limited transport through a bulge or the slow diffusion at a given location induces a bulge. 
We found that loops, which are structures connected to the electrode at both ends, grow significantly faster than individual needles. The formation of features that are connected to the electrode at both ends seems not straightforward. Unfortunately, the initial growth of loops could not be observed. Loops only became visible at later stages of the deposition when the electrode was already covered by lithium needles. Based on the SEM observation in Figure 4a, we assume that loops can form when needles grow almost parallel to the electrode surface and meet an obstacle, e.g. other needles. The tip of the needle and the obstacle can reduce their surface energy by coalescence. Given the high surface-to-volume ratio of these structures and the high homologous temperature, this type of diffusion bonding is plausible. Continued lithium insertion at the base of the needle may then result in a buckling of the structure and the loop becomes visible. The hypothesis of an obstacle-based loop formation is in agreement with the increasing number of loops that occur at later stages of the deposition when the electrode surface is covered by a sufficient number of potential obstacles.

While loops are constrained by being connected to the substrate at both ends, needles can freely elongate and kink since they are connected to the substrate only at one point. The stresses in needles are smaller and consequently their crystalline defects may heal. In loops, growth inevitably induces tensile and compressive stresses, and significant growth causes plastic deformation, for example at kinks. Plastic deformation is based on dislocation motion. During deformation, dislocations interfere with each other and multiply. For example, mobile dislocations can interfere with the immobile dislocations of GBs and thereby continuously generate crystalline defects, which increase the number of insertion sites. Furthermore, mechanical stress is expected to induce defects in the SEI. Both of these effects together can explain the faster growth of a segment of a loop compared to that of an individual needle. Due to the constraints of a loop, the mechanical stresses of all of the kinks are interlinked and insertion into a given kink might also trigger mechanical effects and insertion at distant kinks. This could additionally fuel loop growth. Our observations show that new dominating insertion sites occur in the proximity of rapidly growing segments. It is plausible that the stresses are highest close to the insertion points and that the number of kinks between two insertion sites mitigate the stress on kinks that are farther apart. There is no simple pathway of stress relief in growing loops: Growth induces mechanical stress and this stress enhances insertion. Therefore, the growth of loops may be self-sustaining.

Previously we have observed strongly accelerating growth for lithium bushes, which grow from their inside, during the electrodeposition at higher rates. ${ }^{[15]}$ Growth in all directions can result in coalescence with other lithium deposits, creating bushes, which are 3D-interconnected structures. The geometrical constraints inside a strongly interlinked bush are even more severe than 
the constraints in a loop since the 3D confinement of a bush increases the stiffness in three dimensions and geometrical changes induce additional stresses. This means that insertion into kinks leads to a stronger activation of insertion sites. Since the lithium deposits located at the perimeter of the bush are less constrained, little stress is induced in their GBs and growth is slower. Therefore, the insertion into the GBs is only promoted in the inner part of the bush where the ligaments are strongly interlinked, resulting in the observed growth from the inside of the bushes. This effect of an enhanced insertion at deforming GBs appears to be significant since this growth mode is even dominant while the electrolyte ionically depletes. ${ }^{[15]}$ Models only based on ionic mass transport limitations predict a growth at the tips of the bush. In the experiments, a three-dimensional growth from the inside of the bush was observed. ${ }^{[15]}$ Many publications have shown that large area substrates (e.g. porous metals or carbons) minimize dendritic growth. ${ }^{[41-43]}$ A usual interpretation of this effect is the smaller current density at such substrates, which has been suggested to avoid electrolyte depletion. Since our previous results indicate that depletion is not dominating the growth, ${ }^{[15]}$ we suggest an alternative explanation of the effect: The larger substrate area results in a large substrate-lithium interface, which might provide large number of insertion sites. Furthermore, similar to the preferred insertion inside a bush, the insertion in a porous and stiff substrate can create mechanical stresses due to the limited space, enhancing lithium growth inside the porous structure.

It has been reported by several groups that stack pressure can prevent dendrite formation in lithium metal cells with liquid ${ }^{[17-20]}$ or solid ${ }^{[44]}$ electrolytes. In the context of this work this strategy might work best if it stresses (pressures) above the yield stress of bulk lithium $\left(<1 \mathrm{MPa}^{[45]}\right)$ are maintained throughout a cycle, resulting in continuous plasticity of the electrode and a homogeneous generation of insertion sites. A size-dependent yield strength of lithium was observed for micro pillars ${ }^{[46]}$ and for electrochemically grown lithium whiskers ${ }^{[36]}$. Therefore, small and hence strong features may develop that will not plastically deform due to the stack pressure. Without plasticity, they may not be able to sustain their insertion sites and hence cease growth. Plasticity in large flat regions or in larger features with low yield stress may still be maintained and these regions therefore may bypass the small and strong protrusions during growth.

\section{Conclusion}

Operando light microscopy close to the physical resolution limit of visible light was used to investigate the growth of lithium during electrodeposition. Lithium is inserted into needles and loops at their base and at kinks. EBSD showed that kinks coincide with GBs between lithium segments. The statistically representative data shows that growth of a loop segment can be faster 
than that of a needle. Loops and needles exhibit similar shapes and hence we attribute the observed difference in growth velocity to different mechanical boundary conditions. While needles are freestanding, loops are held at both ends. Consequently, the growth of loops induces mechanical stresses. The observations suggest that electrodeposition of lithium couples to mechanical stress: Electrodeposition not only generates stresses but is also is affected by them. During electrodeposition, plastic deformation of the lithium may generate insertion sites for lithium that can control the growth of a structure. After the deposition of an adatom, surface diffusion and diffusion along GBs are important transport mechanisms: Coupled diffusion along the surface and in a GB moves lithium atoms inside the lithium structures where they are inserted into the crystal. Here the climb of dislocations and the occupation of vacancies act as sinks for atoms and mediate crystal growth. Maintained plasticity can continuously generate these defects and hence can drive the growth of segments. The Burgers vector in bcc lattices is the $\langle 111\rangle$ direction. If climb of dislocations controls growth, the segments will be oriented along this direction.

Following these interpretations, two very different strategies may be used to prevent the nonuniform growth of lithium: (i) Shutting down GB diffusion, either by SEI components that block lithium from entering into the GB or by foreign atoms within the GB blocking lithium transport. (ii) Distribute the insertion sites uniformly and facilitate the growth of a large number lithium grains. This might be accomplished by a uniform stack pressure causing homogeneous plastic deformation.

This work highlights the importance of crystalline defects and mechanical stresses for the growth of lithium. Since sodium and potassium also have low melting points, the suggested growth mechanism can be expected to be also relevant for their deposition.

\section{Experimental}

Lithium was electrodeposited onto a copper electrode, prepared from a $0.5 \mathrm{~mm}$ thick foil (99.9999 \% Puratronic foil from Alfa Aesar), in a liquid electrolyte (1 M LiPF 6 in a 1:1 volume ratio mixture of ethylene carbonate (EC) and dimethyl carbonate (DMC) from Merck). The counter electrode was a piece of lithium (99.9 \% from Alfa Aesar). The operando light microscopy cells were prepared and assembled as described in our previous work. ${ }^{[15]}$ After an electrochemical pretreatment, ${ }^{[15]}$ the copper electrode was covered with a dense film of lithium spheres and lithium was galvanostatically deposited onto this layer at current densities of $-0.05 \mathrm{~mA} \mathrm{~cm}^{-2}$ and $-0.5 \mathrm{~mA} \mathrm{~cm}^{-2}$ with a potentiostat (CompactStat.e, Ivium Technologies B.V.). The operando light microscopy experiments were performed with a Nikon Eclipse LVUDM in bright field using an objective scanning system with a piezo drive (Physik Instrumente 
(PI) $\mathrm{GmbH} \& \mathrm{Co} . \mathrm{KG}$ ) for the fast acquisition of image stacks. We investigate individual structures and hence the images of the full electrode were cropped to sections with these analyzed structures. To increase the image quality, only the images of each acquired $z$-stack that were relevant for these structures were used to calculate the images with an extended depth of field. Images with an extended depth of field were calculated with an algorithm based on Laplacian pyramids. ${ }^{[47]}$ For better visibility, the microscopy images were processed using noise reduction and greyscale adjustments. For the measurement of the length and growth velocity of the lithium structures, the image stacks of the raw images were evaluated to determine the $z$-coordinates of the measured features in addition to their $x$ - and $y$-coordinates. The distance $z$-direction between two images of the stack is $2.2 \mu \mathrm{m}$ and the error in $z$-direction will dominate the total error since the lateral resolution is about $0.5 \mu \mathrm{m}$. Therefore, we expect an uncertainty of the length measurements of needles and segments below $3 \mu \mathrm{m}$. After operando microscopy, the electrodes were examined in the SEM. For that, the cell was disassembled inside an argon-filled glove box $\left(\mathrm{H}_{2} \mathrm{O}\right.$ and $\mathrm{O}_{2}$ content typically $\left.<0.1 \mathrm{ppm}\right)$ and the electrodes were washed in DMC. They were transferred to the SEM using a vacuum transfer system (Leica EM VCT 100).

\section{Acknowledgements}

The author J. Becherer gratefully acknowledges funding by the Friedrich-and-Elisabeth-Boysen Foundation (project number BOY-161).

\section{References}

[1] J. H. Um, S. H. Yu, Adv. Energy Mater. 2020, 11, 2003004.

[2] T. Foroozan, S. Sharifi-Asl, R. Shahbazian-Yassar, J. Power Sources 2020, 461, 228135.

[3] Z. Xie, Z. Wu, X. An, X. Yue, J. Wang, A. Abudula, G. Guan, Energy Storage Mater. 2020, 32, 386.

[4] H. Liu, X.-B. Cheng, Z. Jin, R. Zhang, G. Wang, L.-Q. Chen, Q.-B. Liu, J.-Q. Huang, Q. Zhang, EnergyChem 2019, 1, 100003.

[5] P. Zou, Y. Sui, H. Zhan, C. Wang, H. L. Xin, H. Cheng, F. Kang, C. Yang, Chem. Rev. 2021, 121, 5986.

[6] K. Nishikawa, T. Mori, T. Nishida, Y. Fukunaka, M. Rosso, T. Homma, J. Electrochem. Soc. 2010, 157, A1212.

[7] K. Nishikawa, T. Mori, T. Nishida, Y. Fukunaka, M. Rosso, J. Electroanal. Chem. 2011, 661, 84 .

[8] J. I. Yamaki, S. I. Tobishima, K. Hayashi, K. Saito, Y. Nemoto, M. Arakawa, J. Power Sources 1998, 74, 219. 
[9] O. Crowther, A. C. West, J. Electrochem. Soc. 2008, 155, A806.

[10] P. Bai, J. Li, F. R. Brushett, M. Z. Bazant, Energy Environ. Sci. 2016, 9, 3221.

[11] A. Kushima, K. P. So, C. Su, P. Bai, N. Kuriyama, T. Maebashi, Y. Fujiwara, M. Z. Bazant, J. Li, Nano Energy 2017, 32, 271.

[12] J. Steiger, D. Kramer, R. Mönig, J. Power Sources 2014, 261, 112.

[13] J. Steiger, D. Kramer, R. Mönig, Electrochim. Acta 2014, 136, 529.

[14] J. Steiger, G. Richter, M. Wenk, D. Kramer, R. Mönig, Electrochem. commun. 2015, $50,11$.

[15] J. Becherer, D. Kramer, R. Mönig, ChemElectroChem 2021, 8, 3882.

[16] A. A. Rulev, Y. O. Kondratyeva, L. V. Yashina, D. M. Itkis, J. Phys. Chem. Lett. 2020, $11,10511$.

[17] D. P. Wilkinson, H. Blom, K. Brandt, D. Wainwright, J. Power Sources 1991, 36, 517.

[18] T. Hirai, I. Yoshimatsu, J. Yamaki, J. Electrochem. Soc. 1994, 141, 611.

[19] L. Gireaud, S. Grugeon, S. Laruelle, B. Yrieix, J. M. Tarascon, Electrochem. commun. 2006, $8,1639$.

[20] X. Yin, W. Tang, I. D. Jung, K. C. Phua, S. Adams, S. W. Lee, G. W. Zheng, Nano Energy 2018, 50, 659.

[21] Y. S. Cohen, Y. Cohen, D. Aurbach, J. Phys. Chem. B 2000, 104, 12282.

[22] D. Aurbach, E. Zinigrad, Y. Cohen, H. Teller, Solid State Ionics 2002, 148, 405.

[23] T. Michely, J. Krug, Islands, Mounds and Atoms, Springer-Verlag, Berlin, 2004.

[24] M. Schamel, C. Schopf, D. Linsler, S. T. Haag, L. Hofacker, C. Kappel, H. P. Strunk, G. Richter, Int. J. Mater. Res. 2011, 102, 828.

[25] M. Jäckle, K. Helmbrecht, M. Smits, D. Stottmeister, A. Groß, Energy Environ. Sci. 2018, 11, 3400 .

[26] E. Chason, B. W. Sheldon, L. B. Freund, J. A. Floro, S. J. Hearne, Phys. Rev. Lett. 2002, $88,156103$.

[27] C. W. Pao, S. M. Foiles, E. B. Webb, D. J. Srolovitz, J. A. Floro, Phys. Rev. Lett. 2007, 99, 036102.

[28] J. N. Chazalviel, Phys. Rev. A 1990, 42, 7355.

[29] C. Monroe, J. Newman, J. Electrochem. Soc. 2003, 150, A1377.

[30] P. M. Sargent, M. F. Ashby, Scr. Metall. 1984, 18, 145.

[31] Y. Chen, Z. Wang, X. Li, X. Yao, C. Wang, Y. Li, W. Xue, D. Yu, S. Y. Kim, F. Yang, A. Kushima, G. Zhang, H. Huang, N. Wu, Y. W. Mai, J. B. Goodenough, J. Li, Nature 2020, 578, 251.

[32] D. Weiss, H. Gao, E. Arzt, Acta Mater. 2001, 49, 2395. 
[33] D. Magnfält, G. Abadias, K. Sarakinos, Appl. Phys. Lett. 2013, 103, 4.

[34] Y. Li, Y. Li, A. Pei, K. Yan, Y. Sun, C. L. Wu, L. M. Joubert, R. Chin, A. L. Koh, Y. Yu, J. Perrino, B. Butz, S. Chu, Y. Cui, Science 2017, 358, 506.

[35] T. Yang, H. Li, Y. Tang, J. Chen, H. Ye, B. Wang, Y. Zhang, C. Du, J. Yao, B. Guo, T. Shen, L. Zhang, T. Zhu, J. Huang, Sci. Bull. 2021, 66, 1754.

[36] L. Zhang, T. Yang, C. Du, Q. Liu, Y. Tang, J. Zhao, B. Wang, T. Chen, Y. Sun, P. Jia, H. Li, L. Geng, J. Chen, H. Ye, Z. Wang, Y. Li, H. Sun, X. Li, Q. Dai, Y. Tang, Q. Peng, T. Shen, S. Zhang, T. Zhu, J. Huang, Nat. Nanotechnol. 2020, 15, 94.

[37] Y. He, X. Ren, Y. Xu, M. H. Engelhard, X. Li, J. Xiao, J. Liu, J. G. Zhang, W. Xu, C. Wang, Nat. Nanotechnol. 2019, 14, 1042.

[38] B. Z. Lee, D. N. Lee, Acta Mater. 1998, 46, 3701.

[39] G. Wulff, Zeitschrift für Krist. - Cryst. Mater. 1901, 34, 449.

[40] Y. Zhang, V. Viswanathan, Langmuir 2020, 36, 11450.

[41] Y. X. Zhan, P. Shi, X. Q. Zhang, F. Ding, J. Q. Huang, Z. Jin, R. Xiang, X. Liu, Q. Zhang, Energy Technol. 2021, 9, 2000700.

[42] J. Zheng, M. S. Kim, Z. Tu, S. Choudhury, T. Tang, L. A. Archer, Chem. Soc. Rev. 2020, 49, 2701.

[43] H. Yang, C. Guo, A. Naveed, J. Lei, J. Yang, Y. Nuli, J. Wang, Energy Storage Mater. 2018, 14, 199.

[44] P. Barai, K. Higa, V. Srinivasan, J. Electrochem. Soc. 2018, 165, A2654.

[45] A. Masias, N. Felten, R. Garcia-Mendez, J. Wolfenstine, J. Sakamoto, J. Mater. Sci. 2019, 54, 2585.

[46] C. Xu, Z. Ahmad, A. Aryanfar, V. Viswanathan, J. R. Greer, Proc. Natl. Acad. Sci. U. S. A. 2017, 114, 57.

[47] W. Wang, F. Chang, J. Comput. 2011, 6, 2559. 